VII Congresso Brasileiro de Informática na Educação (CBIE 2018)

Anais do XXIX Simpósio Brasileiro de Informática na Educação (SBIE 2018)

\title{
Um panorama sobre Learning Analytics em Objetos de Aprendizagem
}

\author{
João Pedro Dewes Guterres' ${ }^{1}$, Milene Selbach Silveira' \\ ${ }^{1}$ Escola Politécnica, Programa de Pós-Graduação em Ciência da Computação - Pontifícia \\ Universidade Católica do Rio Grande do Sul (PUCRS) \\ Caixa Postal 90619-900 - Porto Alegre - RS - Brasil \\ joao.guterreseacad.pucrs.br, milene.silveira@pucrs.br
}

\begin{abstract}
Learning Analytics has stood out as an alternative to better understand students' behavior and performance. The new technologies used to develop Learning Objects opens up a possibility of join Learning Analytics to Learning Objects. In order to understand the possibilities of joining them, we performed literature review, and present an overview of techniques, technologies, analytical variables and benefits of its use.
\end{abstract}

Resumo. O uso de técnicas de Learning Analytics tem se destacado como alternativa para compreender melhor o comportamento e a performance dos alunos. Com o uso de novas tecnologias para o desenvolvimento de Objetos de Aprendizagem, abre-se a possibilidade de agregar - a estes - essas técnicas. Para compreender e investigar esse tema, foi realizada uma revisão de literatura, apresentando-se um panorama sobre as técnicas, tecnologias, variáveis analíticas e benefícios do uso de Learning Analytics em objetos de aprendizagem.

\section{Introdução}

O uso da linguagem HTML5 tem sido encorajado na produção de objetos de aprendizagem (OAs) [Braga et al. 2017; Cruz e Rodrigues 2016; Otsuka et al. 2012], substituindo o Flash, que não possui suporte nos dispositivos móveis e browser recentes [Barçante et al. 2017]. O HTML5 é uma linguagem não proprietária, nativa em dispositivos móveis que possui inúmeras APIs (Application Programming Interface conjunto de instruções, rotinas e padrões de programação) que permitem usar dados coletados dos usuários como latitude, aceleração 3D, armazenamento local, acesso nativo a streaming de áudio e vídeo, animações procedurais e declarativas, etc. [Garaizar e Guenaga 2014]. Com estas características, abre-se a possibilidade de integrar a produção de OAs com novas técnicas e recursos, incluindo Learning Analytics, para coletar dados que possam ser utilizados compreender melhor o comportamento e performance dos alunos.

Learning Analytics são um conjunto de técnicas para medir, coletar, analisar e relatar dados sobre estudantes e seu contexto, tendo como propósito entender e otimizar o aprendizado no ambiente de aprendizagem [Siemens et al. 2011]. Segundo Johnson et al. (2011), Learning Analytics referem-se à interpretação de um grande volume de dados produzidos pelos alunos, a fim de avaliar o seu progresso acadêmico, predizer o desempenho e detectar possíveis problemas de aprendizagem. Já OAs são "Quaisquer 
VII Congresso Brasileiro de Informática na Educação (CBIE 2018)

Anais do XXIX Simpósio Brasileiro de Informática na Educação (SBIE 2018)

materiais eletrônicos (como imagens, vídeos, páginas web, animações ou simulações), desde que tragam informações destinadas à construção do conhecimento (conteúdo autocontido), explicitem seus objetivos pedagógicos e estejam estruturados de tal forma que possam ser reutilizados e recombinados com outros objetos de aprendizagem (padronização)" [Carneiro e Silveira 2014].

Atualmente, a utilização de Learning Analytics em OAs ainda é pouco explorada e carece de alternativas que envolvam seu uso fora do escopo de Learning Managment Systems (LMS), que costuma ser limitado à contabilização de acessos desses objetos nos LMS ou em Repositórios de OAs [Júnior et al. 2012; Moissa et al. 2014], ao tempo de acesso aos objetos [Rufino 2017], e ao uso de frameworks para salvar resultados de exercícios [Wu et al. 2015].

Considera-se que a introdução de Learning Analytics em OAs pode potencializar a compreensão sobre o comportamento e performance dos alunos, visto que essas técnicas podem atuar sobre uma menor granularidade dentro de um OA, o que não seria possível se este estivesse empacotado e integrado a um LMS.

Sendo assim, considerando a importância desse tópico para a área de Informática na Educação, é necessário conhecer as técnicas, tecnologias, variáveis analíticas e benefícios do uso de Learning Analytics em OAs. A fim de se construir uma base teórica e científica que permita subsidiar trabalhos futuros neste segmento, são apresentados e discutidos resultados uma revisão sistemática da literatura sobre o tema.

O trabalho está organizado em 4 seções: a seção 2 apresenta a metodologia adotada na revisão sistemática; a seção 3 apresenta os resultados da uma revisão sistemática, buscando responder as questões de pesquisa propostas; e, por fim, a seção 4 apresenta as conclusões deste trabalho, seguidas das referências nele utilizadas.

\section{Metodologia}

Inicialmente, foram realizadas pesquisas para identificar a existência de revisões sistemáticas sobre o uso de Learning Analytics em OAs. No entanto, nenhum artigo específico foi encontrado sobre o assunto. Assim, de modo a compreender o cenário de pesquisa nessa área, foram elaboradas as seguintes questões de pesquisa:

- RQ1: Quais técnicas de Learning Analytics são adotadas em OAs?

- RQ2: Quais tecnologias são utilizadas no desenvolvimento de OAs para apoiar Learning Analytics?

- RQ3: Quais são as variáveis analíticas utilizadas em OAs?

- RQ4: Quais são os benefícios em utilizar Learning Analytics em OAs?

A primeira fase da revisão sistemática, elaborada seguindo o protocolo proposto por Kitchenham (2007), visou capturar respostas para estas questões em bases internacionais (Science Direct, IEEE, Springer, ACM e Scopus). Para isso foi utilizada a seguinte string de busca: "learning analytics" AND "learning objects", resultando num total de 337 publicações e na seleção final de 18 publicações relevantes. Os dados foram coletados em dezembro de 2017 e o processo de análise dos dados foi realizado durante o primero trimestre de 2018, utilizando o software RQDA para codificação dos dados de acordo com as questões de pesquisa. 
VII Congresso Brasileiro de Informática na Educação (CBIE 2018)

Anais do XXIX Simpósio Brasileiro de Informática na Educação (SBIE 2018)

De modo a complementar os resultados da revisão, na segunda fase da pesquisa, buscou-se também investigar as iniciativas de pesquisa sobre o tema no cenário brasileiro e latino-americano, utilizando o mesmo protocolo. Com isso, foram investigadas as bases de dados relacionadas à Comissão Especial de Informática na Educação (http://ceie-br.org/), incluindo os eventos SBIE (Simpósio Brasileiro de Informática na Educação) e WIE (Workshop de Informática na Escola), os Workshops relacionados ao CBIE (Congresso Brasileiro de Informática na Educação), DesafIE (Workshop de Desafios da Computação Aplicada à Educação), além de RENOTE (Revista Novas Tecnologias na Educação) e TISE (International Congress on Educational Informatics $)^{1}$. O resultado dessa seleção encontrou 16 artigos com uso da string de busca 'learning analytics' AND “'objetos de aprendizagem' OR 'learning objects'", resultando na seleção final de mais 3 artigos. Esse segundo processo de busca e seleção foi realizado no mês de junho de 2018 .

\section{Resultados da Revisão Sistemática}

Os resultados da análise realizada serão apresentados a partir de tópicos para responder as questões de pesquisa propostas.

\subsection{Técnicas de Learning Analytics}

O nome das técnicas oriundas da Inteligência de Negócios (Business Intelligence - BI) é bastante heterogêneo, tendo muitas convergências e intersecções entre os conceitos destas técnicas que podem ser utilizadas na área de aprendizagem [Silva et al. 2017]. Buscou-se então, para apresentação de técnicas de Learning Analytics, aquelas que detalham usos específicos dessas técnicas que podem ser adotas em recursos educacionais ou em OAs.

Explicando melhor as diferenças entre os conceitos das técnicas envolvidas, Dyckhoff et al. 2012 citam que técnica de BI se preocupa em fornecer informações para suporte à decisão de gerenciamento, evidenciando um aspecto mais estratégico e menos pedagógico. Já a Análise Acadêmica, segundo Dyckhoff et al. (2012), tem uma perspectiva universitária, incluindo questões organizacionais e financeiras, sendo essa técnica um meio termo entre o aspecto gerencial e o pedagógico. E a Mineração de Dados Educacionais se concentra em ferramentas e métodos para explorar dados provenientes de contextos educacionais, sendo este último voltado para o suporte aos pedagogos e professores [Dyckhoff et al. 2012].

Segundo Dyckhoff et al. (2012), Learning Analytics sintetiza técnicas de diferentes áreas de Mineração de Dados Educacionais, Análise Acadêmica, Análise de Redes Sociais ou Inteligência de Negócios para converter dados educacionais em informações úteis, de modo a promover o aprimoramento do ensino e a aprendizagem. Ogawa et al. (2017) citam que as técnicas de Estatística, e Visualização da Informação (InfoVis) suportam a melhoria do ambiente de aprendizagem por apresentar facilitar a apresentação dos dados de acesso dos OAs. Ampliando esse escopo, Yassine et al. (2016) citam as técnicas de Visualização de dados, Redes Neurais, Análise de Regressão, Análise Web, e Análise das Redes Sociais, abrangendo a união de vários dados oriundos das ferramentas integradas nos ambientes de aprendizagem.

\footnotetext{
1 Não foi possível incluir publicações da conferência LACLO devido a indisponibilidade do site <http://laclo.org/papers> no período de coleta de dados.
} 
VII Congresso Brasileiro de Informática na Educação (CBIE 2018)

Anais do XXIX Simpósio Brasileiro de Informática na Educação (SBIE 2018)

Moissa et al. (2014b) aprofundam a técnica de Análise das Redes Sociais Web, segmentando em dois tipos: $\log _{s}$ Web e Tag de páginas. $\log _{s} W e b$, citado por Moissa et al. (2014b), envolvem o armazenamento de informações do servidor quando os alunos acessam os recursos educacionais, sendo esta técnica menos robusta e de menos precisão, gerando resultados simples como hora de acesso, tempo de sessão, entre outros. Já a Page Tagging (Tag de Páginas), inclui a adição de scripts nas páginas, envolvendo salvamento de informações pelo browser, sendo mais completas e precisas, podendo incluir número de acertos, tempo em cada parte do objeto, etc. [Moissa et al. 2014b]. Contudo, Borba et al. (2016) comenta que a técnica Page Tagging só se aplica a páginas web, o que pode deixar descobertos outros tipos de recursos.

De modo sucinto, Yassine et al. (2016) citam a Web Analytics como uma técnica para medir, coletar e analisar dados quantitativos da Internet relacionados ao comportamento dos usuários em um site, como o rastreamento de cliques, hits, páginas de destino e gostos do usuário. Estes autores e Escobar et al. (2014) citam ferramentas como Google Analytics para esse fim, entretanto, Yassine et al. (2016) destacam ainda o fato dela não ser elaborada para Learning Analytics para uso educacional, evidenciando questões de privacidade que podem ser expostas ao se utilizar ferramentas de uso geral na internet.

Outra técnica mencionada é a Análise de Redes Sociais, que mapeia as relações entre pessoas, grupos e computadores, de modo a monitorar e interpretar as comunicações entre os alunos, professores e recursos educacionais no ambiente de aprendizagem, incluindo neste escopo as ações em fóruns, chats, logs, posts, comentários, etc. [Yassine et al. 2016].

Tendo em vista que muitos desses conceitos são, por vezes, conflitantes e confusos, Silva et al. (2017) elencaram o termo Ciência de Dados Educacionais para englobar todas essas técnicas. Nesse contexto, após estudos realizados, definiu-se pelos autores que a técnica de Mineração de Dados Educacionais foi elencada como a responsável pela análise de dados oriundos de repositórios de OAs, enquanto que se for analisar as diferentes estratégias de aprendizagem usando OAs, esse escopo se insere entre os estudos de Learning Analytics.

\subsection{Tecnologias para inserção das técnicas de Learning Analytics em OAs}

A implementação de OAs contendo técnicas de Learning Analytics pode ser realizada de diversas maneiras, no entanto todas necessitam de uma linguagem de programação server-side (back-end), ou seja, que seja interpretada pelo servidor de aplicação [Borba et al. 2016] e não apenas pelo navegador.

Dyckhoff et al. (2012) citam a possibilidade do desenvolvimento utilizando código server-side .NET integrado com o serviço Windows Communication Foundation (WCF). Neste modelo, as informações são transitadas do OA em .NET por meio do WCF (web services) para uma base de dados, que pode ser remota ou distribuída. Essa comunicação se baseia em mensagens enviadas a cada interação do usuário.

Outra proposta é citada por Ahn et al. (2017), que utiliza um OA em HTML e Javascript e Node.js integrado a um banco de dados Mongoose. Nesse modelo, o Node.js, um script equivalente ao Javascript que roda no lado do servidor, é responsável por realizar a comunicação dos dados do objeto diretamente com o banco de dados. Já Yau e Hristova (2017) propuseram um modelo de serviço em que um OA desenvolvido 
VII Congresso Brasileiro de Informática na Educação (CBIE 2018)

Anais do XXIX Simpósio Brasileiro de Informática na Educação (SBIE 2018)

em Java se comunica com um serviço utilizando uma biblioteca externa JavaScript Object Notation (JSON), responsável converter a resposta do serviço utilizando a linguagem JSON em Java objects, também utilizando o conceito de web services.

Freire et al. (2016) comentam que, para simplificar a integração entre objetos com banco de dados, LMS, por exemplo, é altamente recomendável seguir uma arquitetura orientada a serviços (por exemplo, através de uma API REST que utiliza mecanismos de web services) que não requer intervenção humana, pois automatiza processos e permite uma rápida expansão.

De modo mais simples, Escobar et al. (2014) e Yassine et al. (2016) citam o uso do Google Analytics para gerar dados analíticos de OA. Yassine et al. (2016) também citam o SurfStats Website Traffic Analyzer como outra ferramenta para capturar e salvar dados da sessão de acesso dos usuários. Nesse cenário, não há nenhuma modificação no objeto e os dados coletados são muito genéricos, como o número de visitas e o tempo em que o aluno esteve naquela página, etc., sendo que apenas estes não são ideais para compreender melhor o comportamento dos alunos nos OA.

Para especificar um modelo teórico de uso do Learning Analytics no uso de OAs, Dyckhoff et al. (2012) elaboraram um toolkit denominado eLAT (exploratory Learning Analytics Toolkit), contendo uma arquitetura para auxiliar professores a explorar e correlacionar o uso de conteúdo, propriedades, comportamentos dos usuários, bem como resultados de avaliação com base em indicadores gráficos selecionados individualmente. Nessa arquitetura, os autores utilizaram Sharepoint, Moodle, XML, dois bancos de dados e um sistema web para explorar os dados analíticos. Borba et al. (2016) comenta que o armazenamento pode ser tanto em banco de dados, quanto em arquivos como XML, texto, XLS e CSV.

Krauss et al. (2017) propuseram uma arquitetura LCA (Learning Companion Application), de modo a integrar um repositório de OAs a um LMS. Essa integração é feita por uma interface de comunicação, que possui um sistema de Learning Analytics e de recomendação de OAs. Para isso, os autores utilizaram a especificação da Learning Tools Interoperability Specification (LTI) da IMS [IMS 2015] para comunicação, a qual também é citada por Ochoa e Ternier (2017) para realizar a comunicação de dados analíticos de objetos para um banco de dados. Contudo, são citados também outros padrões da Web, como HTML, JavaScript e web services para essa integração. Já Yassine et al. (2016) mencionam outro framework, o xAPI, como um padrão para rastrear e armazenar as interações dos usuários em OAs.

Wu et al. (2015) relacionam os três frameworks mais conhecidos que permitem a coleta de informações de OAs: DataShop, IMS Caliper e Experience API (xAPI). Entretanto, os autores criticam esses frameworks por eles não trazerem informações suficientes para interpretação completa do perfil do aluno, além de não serem consistentes para o formato de dados dos OAs, prejudicando a interoperabilidade e aumentando custos.

Nesse cenário, observa-se que existem várias alternativas para serem exploradas para realizar a comunicação dos dados obtidos por meio de Learning Analytics dos OAs para uma base de dados. Entretanto, sem haver um padrão específico sobre quais variáveis analíticas são importantes ou que tipos de recursos são abrangidos por essas técnicas, a exploração das técnicas de Learning Analytics ainda é feita de modo experimental, de acordo com as demandas de cada centro de produção. 
VII Congresso Brasileiro de Informática na Educação (CBIE 2018)

Anais do XXIX Simpósio Brasileiro de Informática na Educação (SBIE 2018)

\subsection{Variáveis Analíticas dos OAs}

Tsarmpou e Tambouris (2015) comentam que diferentes tipos de OAs, como, quiz, recursos, jogos, podem possuir diferentes dados sobre as atividades dos usuários. Nessa relação, os autores citam que um objeto puramente teórico pode ter, entre outras, variáveis como número de visitas e tempo dedicado, já um objeto prático pode ter informações adicionais sobre notas, respostas escolhidas, número de tentativas, tentativas concluídas.

Ahn et al. (2017), de modo simplificado, elegeram poucas variáveis de comportamento dos alunos, citando a leitura de conteúdos, tomada de notas e realização de exercícios. De modo análogo, Duval et al. (2011) comentam que é salvo o tempo em que o aluno fica em cada atividade, não citando exemplos de variáveis. Dyckhoff et al. (2012) citam o número de cliques realizados pelo usuário, além de indicar o número de acessos aos recursos. Ao integrar técnicas de Learning Analytics com gamificação, Ogawa et al. (2017) elegeram o andamento do aluno disciplina, englobando as variáveis de progresso e desempenho que permitiram elencar uma pontuação aos alunos.

Escobar et al. (2014) se limitam a explicar as variáveis analíticas propostas pelo Google Analytics, como Goal Starts, Goal Completion (quando usuário completa um determinado objetivo), Goal Completion Location (página redirecionada ao usuário no sucesso de um objetivo), e Goal Abandonment Rate (taxa de abandono do usuário em determinada página), utilizadas para monitorar os caminhos dos usuários pelos sites. Nesse cenário, os autores também enumeram algumas métricas, sendo que é possível sua customização: contador de visitas (geral), contador de visitas (quantos usuários visitaram a página), tempo no site, tempo na página, taxa de retorno a uma página (quantas vezes o usuário retorna a uma mesma página), taxa de saída (quantas vezes o usuário sai em determinada página), número de páginas visitadas por usuário, etc.

Freire et al. (2016) citam cliques no mouse, uso de teclas do teclado, e toques na tela touchscreen como exemplos de dados que também podem ser coletados. Moissa et al. (2014b) também elencam inúmeras variáveis, com destaque a total de visitas por aluno, tempo de acesso individual, frequência de acesso, tipo de browser, tipo de sistema operacional e resolução de tela, entre outros. Van Diepen e Bredeweg (2016), por fim, relacionam as variáveis ao comportamento dos alunos em objetos teóricos e de exercícios, indicando o tempo, número de cliques, número de tentativas bem e mal sucedidas e distribuição de tempo entre exercício e teoria.

A Tabela 1 apresenta um resumo dessas variáveis analisadas, que podem ser divididas entre variáveis próprias do objeto, próprias do aluno e variáveis que podem ser atribuídas tanto para os objetos como para os alunos.

Tabela 1. Resumo das variáveis analíticas.

\begin{tabular}{|l|l|l|}
\hline Variáveis dos OAs & Variáveis do aluno & Variáveis de alunos e OAs \\
\hline
\end{tabular}


VII Congresso Brasileiro de Informática na Educação (CBIE 2018)

Anais do XXIX Simpósio Brasileiro de Informática na Educação (SBIE 2018)

\begin{tabular}{|c|c|c|}
\hline $\begin{array}{l}\text { - Número de visitas } \\
\text { - Notas dos alunos } \\
\text { (média) } \\
\text { - } \text { Taxa de saída em } \\
\text { cada atividade } \\
\text { - } \begin{array}{l}\text { Taxa de retorno ao } \\
\text { objeto }\end{array}\end{array}$ & $\begin{array}{l}\text { - Número de conteudos lidos } \\
\text { - Desempenho do aluno/notas } \\
\text { - Número de objetos } \\
\text { acessados ou progresso } \\
\text { - } \text { Distribuição de tempo entre } \\
\text { exercício e teoria } \\
\text { - } \\
\text { Tipo de browser, sistema } \\
\text { operacional e resolução de } \\
\text { tela }\end{array}$ & $\begin{array}{l}\text { - Número de cliques } \\
\text { - Tempo em cada } \\
\text { atividade } \\
\text { - Número de tentativas } \\
\text { bem-sucedidas e mal } \\
\text { sucedidas }\end{array}$ \\
\hline
\end{tabular}

\subsection{Benefícios do uso de Learning Analitics em OAs}

Krauss et al. (2017) citam a identificação de fraquezas de aprendizagem para melhorar o ensino com a ajuda de um módulo de análise de aprendizagem, utilizando os dados provindos dos objetos. Nesse cenário, os autores citam a possibilidade de um sistema de recomendação se adaptar de acordo com o comportamento do aluno, citando, como exemplo, a previsão de OAs apropriados que se encaixem nas necessidades dos usuários. O'Riordan et al. (2015) comentam que a análise das interações dos alunos é reconhecida como uma valiosa ferramenta para prover feedback aos alunos.

Tsarmpou e Tambouris (2015) citam que as técnicas de visualização de dados analíticos ajudam os instrutores a identificar quais estudantes que estão em risco e permitem uma intervenção para reduzir a possibilidade de falha ou reprovação dos alunos. Nesse cenário, podem ser oferecidos aos alunos exercícios adicionais e revisões. Freire et al. (2016) relatam que esses dados podem ser utilizados para adaptar o conteúdo, apresentação e navegação, de acordo com o perfil do aluno.

Do mesmo modo, o próprio aluno pode se beneficiar dos dados que são obtidos a partir do uso dos objetos, indicando informações sobre seu progresso e seu desempenho de aprendizagem [Van Diepen e Bredeweg 2016]. Yau e Hristova (2017) comentam que o uso desses dados pode ser uma maneira de motivar os alunos a aprimorarem sua aprendizagem atual, contínua e futura, mensurando seu progresso, conquistas, pontos fortes e fracos. Além disso, pode-se usar a análise preditiva para fazer intervenções e para ajudar a reduzir as taxas de abandono escolar dos alunos.

Analisando por outra perspectiva, Tsarmpou e Tambouris (2015) relatam que os feedbacks oriundos do Learning Analytics também podem ser utilizados para avaliar o próprio OA, provendo informações que podem levar ao reforço de determinado conteúdo ou melhoria na qualidade do objeto. Esse fato também é mencionado por Yassine et al. (2016), ao mencionarem que os dados fornecem indicações da qualidade dos objetos para realizar a melhoria contínua nos recursos. Estes autores também citam que a obtenção dos dados analíticos permite identificar popularidade e tendências, evidenciando uma melhor percepção sobre as preferências dos alunos como tipos de recursos, abordagens, estilos de aprendizagem.

Outro benefício inclui o refinamento das trajetórias de aprendizagem, que poderiam ser melhor exploradas se pudessem estar dentro dos OAs. Atualmente, as trajetórias são feitas a partir de um grafo de atividade, para identificar as páginas acessadas e entender o comportamento do aluno; ou grafos com as sequências de páginas mais frequentes, para entender como os alunos navegam pela disciplina [Borba et al. 2016]. Contudo, no modelo tracicional, tendo o objeto encapsulado, ao adotar essa 
VII Congresso Brasileiro de Informática na Educação (CBIE 2018)

Anais do XXIX Simpósio Brasileiro de Informática na Educação (SBIE 2018)

estratégia, se um OA estiver em apenas uma página HTML, não é possível compreender os detalhes desse comportamento interno.

\section{Conclusões}

Nessa pesquisa, buscou-se investigar o panorama atual sobre Learning Analytics aplicados em OAs, exibindo e discutindo as técnicas, tecnologias, variáveis analíticas e benefícios de seu uso, apresentando um esforço inicial dessa pesquisa em divulgar e incentivar o uso dessas técnicas.

As técnicas apresentam um escopo amplo do que pode ser implementado, principalmente se integradas com outros ambientes de aprendizagem. Nas tecnologias, são apontadas linguagens server-side e frameworks recentes que possuem um grande potencial, porém ainda são implementados apenas parcialmente em OAs. São apresentadas, também, uma série de variáveis que podem ser usadas para compreender o comportamento do usuário. E, por fim, os benefícios do uso de Learning Analytics em OAs apontam discussões e exemplos de melhoria pelo uso dessas técnicas.

A adoção dessas técnicas na produção de OAs pode instaurar um novo paradigma nessa área, potencializando e expandindo o processo de compreensão do comportamento e performance do aluno pelo uso do objeto, bem como ajudar na avaliação dos próprios OAs. Como próximos passos desta pesquisa, pretende-se explorar alternativas de aplicação de Learning Analytics e realizar experimentos para sua adaptação à produção de OAs, verificando, na prática, as ideias aqui apresentadas e discutidas.

\section{Agradecimentos}

João Pedro Dewes Guterres agradece ao CNPq pela bolsa de estudos no Doutorado. O presente artigo foi alcançado em cooperação com a Hewlett Packard Brasil LTDA. e com recursos provenientes da Lei de Informática (Lei $n^{\circ} 8.248$, de 1991). O presente trabalho foi realizado com apoio da Coordenação de Aperfeiçoamento de Pessoal Nivel Superior - Brasil (CAPES) - Código de Financiamento 001.

\section{Referências}

Ahn, J.Y.; Mun, G.S.; Han, K.S.; Choi, S.H. (2017) "An online authoring tool for creating activity-based learning objects". In: Education and Information Technologies, vol. 22(6), pp. 3005-3015.

Barçante, M., Oliveira, L. S.; Silveira Ribeiro, M. M. (2017) "Sistema de Informação para avaliação de proficiência de língua estrangeira: Relato de sua evolução tecnológica." In Revista CBTecLE, vol. 1(1), pp. 596-613.

Borba, E.J.; Gasparini, I.; Lichtnow, D.; Pimenta, M.S.; Oliveira, J.P.M. (2016) "Captura e Visualização da Trajetória de Aprendizagem do Aluno: um Mapeamento Sistemático". In: TISE - Nuevas Ideas en Informática Educativa, vol. 12, pp. 105-111.

Braga, J.C.; Vaz, P.; Pimentel, E.; Stiubiener, I. (2017) "Problem Mapping and Recommendations for Accessible Distance Learning for Students with Visual Disabilities". In: Simpósio Brasileiro de Informática na Educação, vol. 28(1), pp. 1077-1087. 
VII Congresso Brasileiro de Informática na Educação (CBIE 2018)

Anais do XXIX Simpósio Brasileiro de Informática na Educação (SBIE 2018)

Carneiro, M.L.F.; Silveira, M.S. (2014) "Objetos de Aprendizagem como elementos facilitadores na Educação a Distância”. In Educar em Revista, vol. 4, pp. 235-260.

Cruz, J.; Rodrigues, W. (2016) "Objetos de Aprendizagem Moveis Uma análise de requisitos funcionais para auxiliar os desenvolvedores". In: Simpósio Brasileiro de Informática na Educação, vol. 27(1), pp. 150-158.

Duval, E.; Verbert, K.; Klerkx, J. (2011) "Towards an open learning infrastructure for open educational resources: Abundance as a platform for innovation, Rainbow of computer science”. In: Rainbow of Computer Science. Berlin: Springer, pp. 144-156.

Dyckhoff, A.L.; Zielke, D.; Bültmann, M.; Chatti, M.A.; Schroeder, U. (2012) "Design and Implementation of a Learning Analytics Toolkit for Teachers". In Educational Technology \& Society, vol. 15(3), pp. 58-76.

Escobar, A.E.; Reyes, P.; Van Hilst, M. (2014) "Metrics for effectiveness of e-learning objects in software engineering education". In: SoutheastCon, pp. 1-5.

Freire, M.; Serrano-Laguna, Á.; Iglesias, B.M.; Martínez-Ortiz, I.; Moreno-Ger, P.; Fernández-Manjón, B. (2016) "Game Learning Analytics: Learning Analytics for Serious Games”, pp 1-29. In: Learning, Design, and Technology. Springer: Cham.

Garaizar, P.; Guenaga, M. (2014) "A Multimodal Learning Analytics View of HTML5 APIs: Technical Benefits and Privacy Risks". In: International Conference on Technological Ecosystems for Enhancing Multiculturality, vol. 2, pp. 275-281.

IMS LTI (2015) “Learning Tools Interoperability Specification”. Relatório Técnico, IMS Global Learning Consortium, v 1.3. Disponível em $<$ https://www.imsglobal.org/activity/learning-tools-interoperability>. Acessado em 21/02/2018.

Johnson, L.; Smith, R.; Willis, H.; Levine, A.; Haywood, K. (2011) "The NMC Horizon Report: 2011 Higher Education Edition”. Relatório Técnico, New Media Consortium, 40p.

Júnior, L.J.; Neto, F.M.M.; Flores, C.D.; Silva, L.C.N.; Sombra, E.L.; Costa, A.A.L. (2012) "Uma extensão do Moodle para recomendação ubíqua de objetos de aprendizagem." In RENOTE, vol. 10(3), 11p.

Kitchenham, B.A. (2007) "Guidelines for performing systematic literature reviews in software engineering”. In: Relatório Técnico, EBSE 2007-001, Ver. 2.3, 44p.

Krauss, C.; Merceron, A.; An, T.S.; Zwicklbauer, M.; Steglich, S.; Arbanowski, S. (2017) "Teaching Advanced Web Technologies with a Mobile Learning Companion Application". In: World Conference on Mobile and Contextual Learning, vol. 16, 4p.

Moissa, B.; Borba, E.J.; Kemczinski, A.; Gasparini, I. (2014) "Uma ferramenta de Visualização da Informação para analisar o comportamento do aluno em um ambiente e-learning e sua trajetória de aprendizagem." In InfoDesign-Revista Brasileira de Design da Informação, vol. 11(3), pp. 337-351.

Moissa, B.; Carvalho, L.S.; Gasparini, I. (2014b) "A Web Analytics and Visualization Tool to Understand Students' Behavior in an Adaptive E-Learning System". In: International Conference on Learning and Collaboration Technologies, vol. 1, pp. 312321. 
VII Congresso Brasileiro de Informática na Educação (CBIE 2018)

Anais do XXIX Simpósio Brasileiro de Informática na Educação (SBIE 2018)

Ochoa, X.; Ternier, S. (2017) "Technical Learning Infrastructure, Interoperability and Standards, Technology Enhanced Learning”. Cham: Springer. pp. 145-155.

Ogawa, A.N.; Klock, A.C.T.; Gasparini, I. (2017) "Integrando Técnicas de Learning Analytics no processo de Gamificação em um Ambiente Virtual de Aprendizagem”. In: Simpósio Brasileiro de Informática na Educação, vol. 28, pp. 615-624.

Otsuka, J.; Beder, D. M.; Montanaro, P.; Rocca, I.; Ghelardi, A. (2012) "Desenvolvimento de jogos educacionais abertos". In: Workshops do Congresso Brasileiro de Informática na Educação, vol. 1(1), 10p.

Rufino, S. (2017) "Arquitetura de comunicação entre AVAs e objetos de aprendizagem dinâmicos utilizando a especificação IMS LTI". Dissertação de Mestrado Profissional, Programa de Pós-Graduação em Engenharia de Software, 101p.

Siemens, G.; Gasevic, D.; Haythornthwaite, C.; Dawson, S.; Shum, S.; Ferguson, R.; Duval, E.; Verbert, K.. Baker, R. (2011) "Open Learning Analytics: an integrated \& modularized platform". In Society for Learning Analytics Research, 20p.

Silva, L.A.; Silveira, I.F.; Silva, L.; Rodrigues, R.; Ramos, J.L.C. (2017) "Ciência de Dados Educacionais: definições e convergências entre as áreas de pesquisa". In: Workshops do Congresso Brasileiro de Informática na Educação, vol. 6, pp. 764-773.

Tsarmpou, P.; Tambouris, E. (2015) "Using learning analytics to enhance UML use case diagrams assimilation in a distance education course". In International Journal of Learning Technology, vol. 10(4), pp. 274-290.

van Diepen, P.; Bredeweg, B. (2016) "Performance Indicators for Online Secondary Education: A Case Study". In: Benelux Conference on Artificial Intelligence, vol. 28, pp. 169-177.

Wu, Y.; Zhong, W.; Zhou, C.; Ma, X. (2015) "Construction on Learning Analytics Object for Sharing and Interoperation of Educational Big Data". In: International Conference on Web-Based Learning, vol. 14, pp. 186-195.

Yassine, S.; Kadry, S.; Sicilia, M. A. (2016) "Learning Analytics and Learning Objects Repositories: overview and future direction". In: Learning, Design, and Technology: an international compendium of theory, research, practice, and policy. Cham: Springer, pp. 1-29.

Yau, J.Y.K.; Hristova, Z. (2017) "Evaluation of an Extendable Context-Aware 'Learning Java' App with Personalized User Profiling". In Technology, knowledge and learning, vol. 22, pp. 1-16. 